\title{
Influencia de variables de diseño en las propiedades mecánicas de una base estabilizada con asfalto espumado
}

\section{Influence of design variables on the mechanical properties of a stabilized base with foamed asphalt}

\section{Fidel García Hernández}

Universidad Autónoma de Chihuahua

fidel@dicym.uson.mx

\section{Horacio Delgado Alamilla}

Instituto Mexicano del Transporte

hdelgado@imt.mx

\author{
Domingo Eduardo Campos Hernández \\ Universidad Autónoma de Querétaro \\ domingoeduardoc@gmail.com
}

Fecha de recepción: 22 de febrero de 2018 / Fecha de aprobación: 26 de abril de 2018

\section{RESUMEN}

La construcción y conservación de nuevas carreteras requiere de muchos recursos tanto naturales como económicos, ya que el reciclado de materiales es una de las técnicas más utilizadas en la actualidad, por lo cual es importante conocer las propiedades mecánicas del tipo de aplicación a utilizar.

El presente trabajo se enfoca en la evaluación de las propiedades mecánicas de una base estabilizada con asfalto espumado (reciclado en frio). El estudio analizó la influencia de dos variables de diseño como son el tipo de filler activo (cal y cemento portland) y el contenido de asfalto espumado (2.2 a 2.4\%). Los parámetros utilizados en la evaluación fueron la resistencia a la tensión indirecta, el ángulo de fricción interna y cohesión (ensayo triaxial) y el módulo resiliente.

Los resultados muestran que la inclusión de cal mejora las propiedades mecánicas de la base estabilizada. En lo referente a los parámetros mecánicos se observó que las mezclas estabilizadas con asfalto espumado presentan un aumento de su resistencia en comparación con una base granular tradicional, siendo la pérdida de cohesión el modo de falla de este tipo de estabilización.

PALABRAS ClAVES: Asfalto espumado, tensión indirecta, base estabilizada, ensayo triaxial.

\section{ABSTRACT}

The construction and conservation of new roads requires many natural and economic resources, so the recycling is one of the most used techniques currently, so it is important to understand the mechanical properties of the type of application to be used.

The present paper focuses on the evaluation of the mechanical properties of a stabilized base with foamed asphalt (cold recycled). The study analyzed the influence of two design variables such as the type of active filler (lime and cement) and the content of foamed asphalt (2.2 to 2.4\%). The parameters used in the evaluation were indirect tensile strength, friction angle and cohesion (triaxial test) and resilient modulus (MR).

The results indicate that the addition of lime improves the mechanical properties of the stabilized base. Regarding mechanical parameters, it was observed that the stabilized mixtures with foamed asphalt show an increase in their resistance compared to a granular base, being the loss of cohesion the failure mode of this type of stabilization.

KEY WORDS: Foamed asphalt, tensile strength, stabilized bases, triaxial test, resilience module. 


\section{INTRODUCCIÓN}

El asfalto espumado se realiza mediante la inyección de una pequeña cantidad de agua fría con aire comprimido sobre asfalto caliente, el intercambio de calor espontaneo deriva en un fenómeno físico que espuma al asfalto. Este proceso altera temporalmente las propiedades físicas del asfalto, ya que el asfalto caliente entra en contacto con el agua fría, provocando que este último se convierta en vapor, el cual es atrapado por miles de pequeñas burbujas de asfalto. Este proceso reduce considerablemente la viscosidad del asfalto y aumenta sus propiedades adherentes lo que posibilita el mezclado con materiales fríos y húmedos.

Se ha observado que el asfalto espumado se distribuye principalmente hacia las partículas finas, por lo que; usualmente el contenido de asfalto se utiliza en el rango del 2-5\% y se encuentra en función del contenido de finos del material a estabilizar (Marek y Anna 2013), (Saleh 2007) y al utilizar una alta cantidad de asfalto propicia una mayor flexibilidad en las mezclas, pero una menor resistencia a la deformación permanente (Jenkins et al., 2007).

En lo referente a la estructura granulométrica, los finos son necesarios para una mejor dispersión en una estabilización con asfalto espumado; sin embargo, el exceso de ellos reduce la permeabilidad y la capacidad de drenaje, lo cual disminuye las propiedades de una base hidráulica (Dal Ben y Jenkins 2014). De igual forma, al elaborar una estabilización con asfalto espumado es crucial vigilar los contenidos de humedad de los materiales, ya que los agregados húmedos propician a una inadecuada dispersión del asfalto y por consiguiente mala unión de estos; lo que conlleva a un impacto en cuanto al daño inducido por humedad, así como una reducción de la durabilidad a largo plazo (Abbas et al., 2013).

La estabilización mediante asfalto espumado tiene como propósito construir una base de alta calidad mediante la reutilización de los materiales existentes en la estructura del pavimento. El asfalto espumado crea una unión discontinua en el material granular, mejorando tanto su cohesión como su susceptibilidad a la humedad (Wirtgen, 2012), con respecto a las propiedades mecánicas de este tipo de mezclas los manuales de diseño de Wirtgen y Australian Roads (Wirtgen, 2012), (Marek y Anna 2013), mencionan que uno de los beneficios que se obtiene es el incremento de la Cohesión (C) sin afectar significativamente el Ángulo de Fricción $(\phi)$. En lo referente a la tensión indirecta en condición seca y saturada se ha concluido por Marek y Anna (2013) que al incrementar el contenido de asfalto espumado la mezcla es menos susceptible a la humedad.

\section{DISEÑO DE UNA BASE ESTABILIZADA CON ASFALTO ESPUMADO}

El diseño de una base estabilizada con asfalto espumado fue realizado siguiendo el esquema descrito en la Figura 1.

\section{MATERIAL ASFÁLTICO}

NIVELES DE DISEÑO

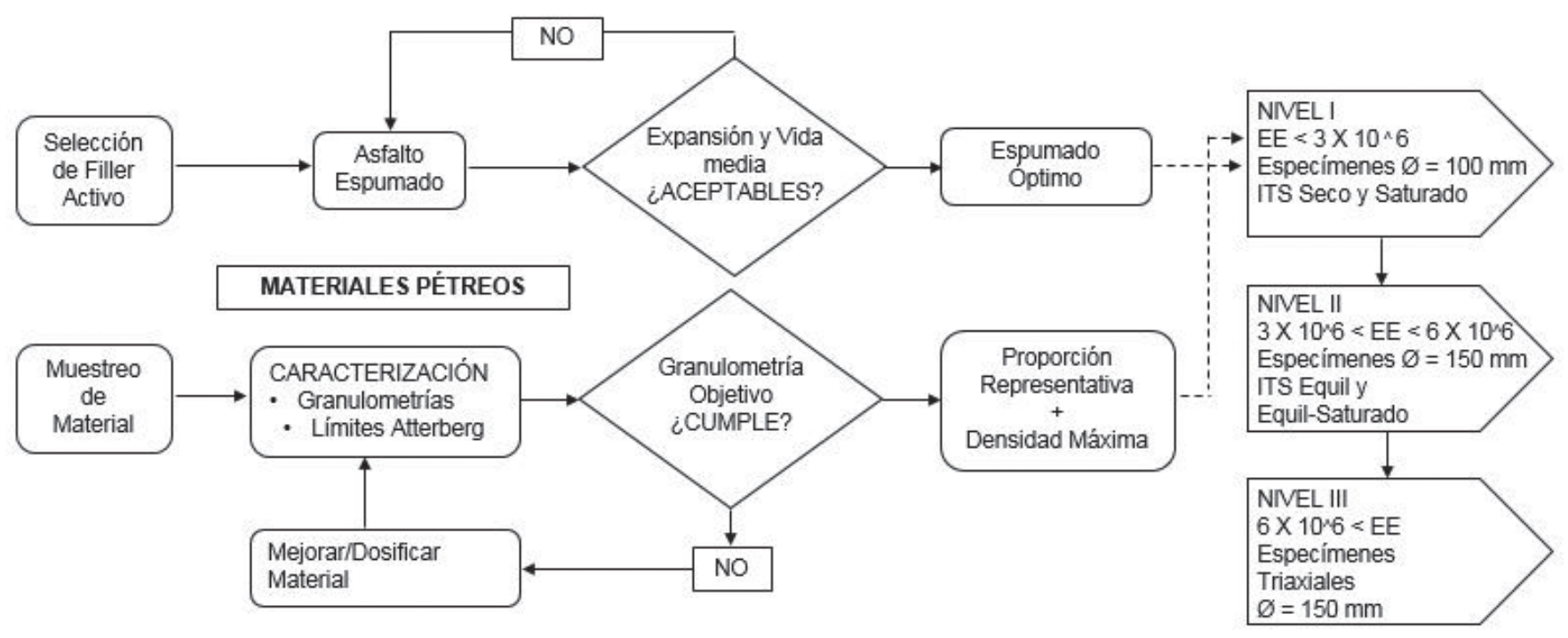

Figura 1. Diagrama de flujo para bases estabilizadas con asfalto espumado 


\section{Caracterización Del Material}

El presente estudio fue realizado con un material recuperado combinado de Carpeta asfáltica-Base granular (50\% RAP y 50\% Base hidráulica), el cual fue mezclado con un material de Banco (material virgen) con calidad de base hidráulica de Tamaño Nominal (TN) de $1 \frac{1}{2} 2$ ". El material de RAP + BASE tuvo un Límite Líquido (LL) de 23.6\% y un Índice de Plasticidad (IP) de 5.2\%. Para el caso del material de banco los valores fueron inapreciables, por lo cual el material no necesita un pretratamiento inicial (adición de cal). El material asfáltico empleado en el estudio fue clasificado como un PG 64-16, al cual no se le adicionó ningún aditivo o polímero.

Estos materiales fueron mezclados en una proporción de $80 \%$ de material RAP + BASE y $20 \%$ de material de Banco. La granulometría obtenida al igual que los límites granulométricos se presentan en la Figura 2, es importante mencionar que son los límites granulométricos los que definen el máximo porcentaje de RAP a utilizar, debido a que la granulometría que se obtiene con el fresado generalmente no cumple con la especificación, por lo cual se tiene que utilizar material pétreo de aporte.

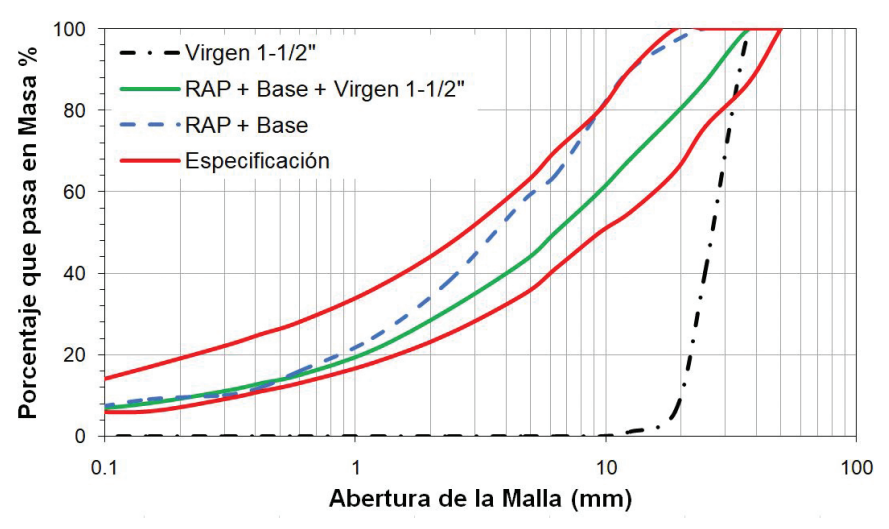

Figura 2. Límites granulométricos para bases espumadas.

Después del mezclado se determinó el Peso Volumétrico Seco Máximo (PVSM, $\gamma_{\mathrm{d}}$ ) mediante la norma AASHTO T-180. La compactación se realiza en cinco (5) capas, aplicando cincuenta y seis (56) golpes por capa, con un pisón de $4.54 \mathrm{~kg}$ y una altura de caída de $45.7 \mathrm{~cm}$ en un molde de $152.4 \mathrm{~mm}$ de diámetro interior. Los resultados obtenidos fueron de $8 \%$ de contenido óptimo de humedad y un PVSM de $2080 \mathrm{~kg} / \mathrm{m}^{3}$.

\section{Propiedades Espumantes Del Asfalto}

Este punto tiene como objetivo determinar el porcentaje de agua, así como la temperatura óptima del asfalto que se requiere para producir las mejores propiedades espumantes de un asfalto, con el propósito de obtener una mayor área de contacto y un mejor revestimiento de los agregados. La determinación se realiza mediante dos parámetros: Relación de expansión $\boldsymbol{R} \boldsymbol{e}$ (medida de la viscosidad del asfalto espumado) y vida media $\tau^{1} \frac{1}{2}$ (medida de la estabilidad del asfalto espumado). Para encontrar los valores de $\boldsymbol{R} \boldsymbol{e}$ y $\mathrm{T}^{1 / 2}, 2$, se procede a realizar un barrido de temperaturas $\left(160^{\circ} \mathrm{C}\right.$, $170{ }^{\circ} \mathrm{C}$ y $180{ }^{\circ} \mathrm{C}$ ) y de contenidos de agua (2\%, 3\% y $4 \%$ ). La Figura 3 presenta la evaluación a $170{ }^{\circ} \mathrm{C}$ (temperatura óptima para el espumado), así como los requerimientos de aceptación y los valores obtenidos para cada temperatura.

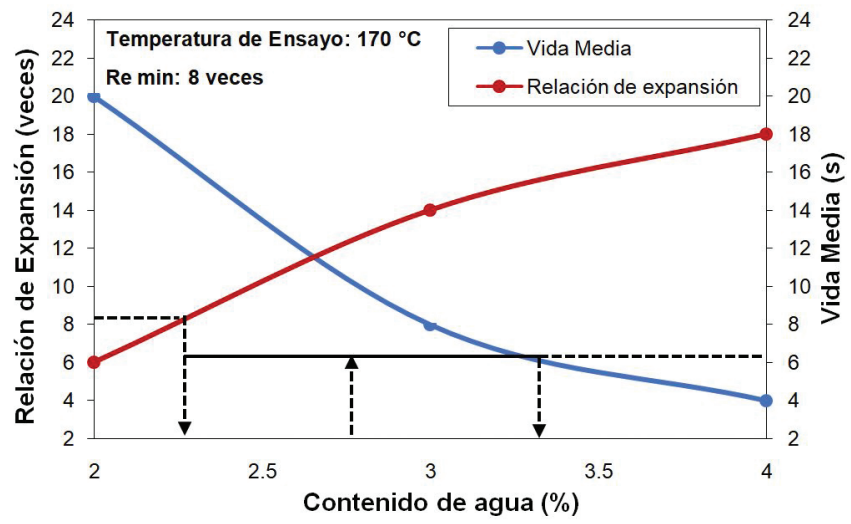

\begin{tabular}{|c|c|c|c|}
\hline $\begin{array}{c}\text { TEMPERATURA DE } \\
\text { PRUEBA }\left({ }^{\circ} \mathrm{C}\right)\end{array}$ & $\begin{array}{c}\text { CANTIDAD DE } \\
\text { AGUA (\%) }\end{array}$ & $\begin{array}{c}\text { VIDA MEDIA } \\
\text { (SEC) }\end{array}$ & $\begin{array}{c}\text { RELACIÓN DE } \\
\text { EXPANSIÓN } \\
\text { (VECES) }\end{array}$ \\
\hline \multirow{2}{*}{$\begin{array}{c}160 \\
170\end{array}$} & 2.7 & 8.5 & 9.8 \\
\cline { 2 - 4 } 180 & 2.7 & 10.5 & 12.2 \\
\cline { 2 - 4 } & 2.7 & 8.5 & 9.5 \\
\hline
\end{tabular}

Figura 3. Determinación de propiedades espumantes a $170^{\circ} \mathrm{C}$ y valores obtenidos a 3 temperaturas

\section{DISEÑO DE LA MEZCLA DE MATERIALES (FÓRMULA DE TRABAJO)}

La metodología para el diseño de la base estabilizada con asfalto espumado considera tres niveles de diseño, los cuales están definidos de acuerdo con el tránsito al cual va a estar sometido el pavimento asfáltico.

\section{Nivel I: Necesidad De Filler Activo}

El presente nivel de diseño tiene como objetivo establecer si la mezcla de materiales (Base hidráulica - RAP) necesita la inclusión de un filler activo. Para esta determinación se realizará un ensayo de susceptibilidad a la humedad mediante el ensayo de Tensile Strength Ratio (TSR, por sus siglas en inglés). Los dos fillers activos utilizados para este tipo de material es la cal y el cemento Portland en porcentajes de $1 \%$. Por lo cual, se realiza 
una evaluación con cada una de las tres variables (Sin filler, 1\% de cal y $1 \%$ de cemento). Todas las mezclas fueron realizadas con $2.4 \%$ de asfalto espumado, el cual es el valor recomendado por la metodología para granulometrías con $<50 \%$ de material que pasa la malla de $4.75 \mathrm{~mm}$ con porcentajes entre 7 y 10 de material que pasa la malla de $0.075 \mathrm{~mm}$ (Wirtgen, 2012). Las probetas fueron elaboradas a un PVSM de $2080 \mathrm{~kg} / \mathrm{m}^{3}$ y una humedad de $8 \%$.

La mezcla sin filler activo obtuvo un valor de TSR en seco de 219 kPa y no obtuvo resistencia en condición saturada, por lo cual no cumple con los requisitos mínimos. En la evaluación en seco de las dos mezclas con filler activo, presentada en la Figura 4, se puede observar que ambas cumplen con el requisito mínimo establecido en la metodología. Sin embargo, en condición saturada la mezcla con cemento portland pierde considerablemente su resistencia, por lo cual se considera inadecuada. La mezcla de materiales con cal también tuvo una reducción de su resistencia en condición saturada pero menos significativa, por lo cual se considera que este filler activo es el más adecuado para la mezcla de materiales propuesta.

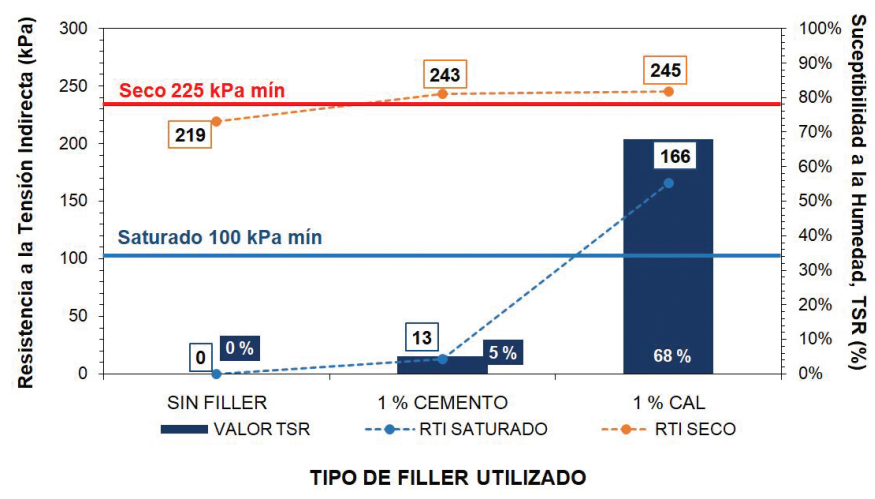

Figura 4. Susceptibilidad a la humedad para diferentes tipos de fillers activos, Nivel I

\section{Nivel II: Contenido De Asfalto Espumado}

En este nivel, se pretende determinar el contenido óptimo de asfalto espumado que requiere el material. Una vez seleccionada una de las tres variantes analizadas en el Nivel I, se realiza un barrido de contenidos de asfalto espumado (4 porcentajes), determinando el mejor desempeño mediante el ensayo de susceptibilidad a la humedad. Las probetas fueron elaboradas a un PVSM de $2080 \mathrm{~kg} / \mathrm{m}^{3}$ y una humedad de $8 \%$. Los resultados presentados en la Figura 5 indican que solo la mezcla con 2.2 de asfalto espumado no cumple con la especificación establecida en la metodología. Se considera que la dispersión de los resultados es de $5 \%$, por lo cual el valor óptimo de espumado será $2.4 \%$ (Primer valor aceptable *1.05). Esta evaluación determina la fórmula de trabajo de la base hidráulica estabilizada con asfalto espumado.

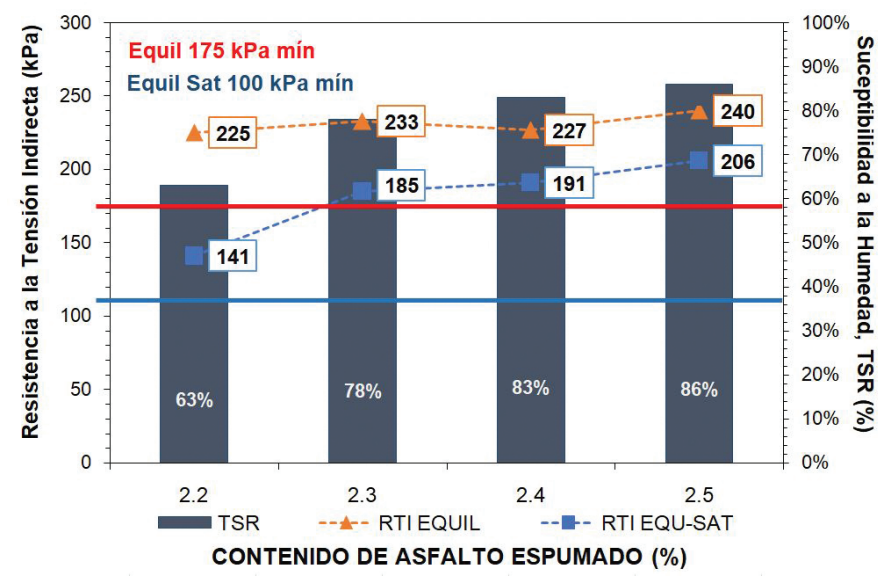

Figura 5. Contenido óptimo de asfalto con 1\% de cal, Nivel II.

De estas dos primeras evaluaciones se observa que a pesar de que todos los valores de $\mathrm{RTI}_{\mathrm{SECO}} / \mathrm{RTI}_{\mathrm{EQUIL}}$ son de magnitudes similares, las propiedades en condición saturada son diferentes. Por lo cual, se puede concluir que el indicador clave en el diseño Nivel I y II es la resistencia de la mezcla condición saturada, lo cual se debe tomar en cuenta tanto en el diseño como en el control de calidad de la base estabilizada con asfalto espumado, ya que uno de los principios del diseño es asegurar la cohesión de la mezcla con asfalto espumado en condiciones de humedad.

\section{Nivel III: Propiedades Mecánicas}

Este nivel tiene como objetivo evaluar las propiedades mecánicas de la mezcla de materiales definida en los dos niveles previos. Los parámetros por evaluar son el valor de cohesión y el ángulo de fricción interna, los cuales serán determinados mediante un ensayo de triaxial. Para esta evaluación se fabricaron 8 especímenes de ensayo y se evaluaron cuatro condiciones de confinamiento ( 0 $\mathrm{kPa}, 50 \mathrm{kPa}, 100 \mathrm{kPa}$ y $200 \mathrm{kPa})$.

La determinación de los valores de cohesión (C) y del ángulo de fricción $(\phi)$ se realizó mediante el Círculo de Mohr- Coulomb. En esta evaluación se obtuvo una cohesión promedio de $248 \mathrm{kPa}$, la cual es ligeramente inferior al valor mínimo $250 \mathrm{kPa}$, y un ángulo de fricción promedio de $45^{\circ}$ (Mínimo 40) (Wirtgen, 2012). Las probetas fueron elaboradas a un PVSM de $2080 \mathrm{~kg} / \mathrm{m}^{3}$ y una humedad de $8 \%$.

\section{INDICADOR PARA EL DISEÑO ESTRUCTURAL DE PAVIMENTOS}

Una de las dudas fundamentales que se tienen al utilizar esta metodología es la capacidad estructural que pueden obtener las bases estabilizadas con asfalto espumado. Por lo cual, fue necesario realizar una evaluación del $\mathrm{M}_{\mathrm{R}}$ (Modulo Resiliente) de la base estabilizada. 
Debido a que el $M_{R}$ no es un valor único; sino que éste depende del confinamiento y la carga axial aplicada al espécimen se optó por graficar el valor de $M_{R}$ medido vs. Esfuerzo volumétrico $(\theta)$, donde:

$\theta=\left(\sigma_{1}+\sigma_{2}+\sigma_{3}\right)$

Donde $\theta$ es el esfuerzo volumétrico, $\sigma_{1}=$ Esfuerzo principal mayor, $\sigma_{2}=$ Esfuerzo principal intermedio (igual a $\sigma_{3}$ para la prueba de $M_{R}$ en el espécimen cilíndrico), $\sigma_{3}=$ Esfuerzo principal menor.

La Figura 6 presenta los resultados obtenidos en la evaluación del $\mathrm{M}_{\mathrm{R}}$ en probetas de $150 \mathrm{~mm}$ de diámetro y $300 \mathrm{~mm}$ de altura, la secuencia de evaluación de acuerdo a NCHRP 01-28A: Pre-acondicionamiento de 1000 repeticiones de carga $\sigma_{1 \max }=227.7 \mathrm{kPa}(33$ psi) y esfuerzo cíclico de $207 \mathrm{kPa}$ (30 psi) de una onda haversiana (carga $0.1 \mathrm{~s}$ y reposo $0.9 \mathrm{~s}$ ), posteriormente se aplican 30 secuencias variando la carga axial y la presión de confinamiento (20.7, 41.4, 69, 103.5 y $138 \mathrm{kPa}$ ). La Figura 6 presenta los resultados obtenidos en el ensayo de módulo resiliente para tres diferentes materiales: Base hidráulica convencional, Base con material reciclado $2.4 \%$ de asfalto espumado y $1 \%$ de cal, Base con material reciclado $0 \%$ de asfalto espumado y $1 \%$ de cal. Las probetas fueron elaboradas a un PVSM de $2080 \mathrm{~kg} / \mathrm{m}^{3}$ y una humedad de $8 \%$.

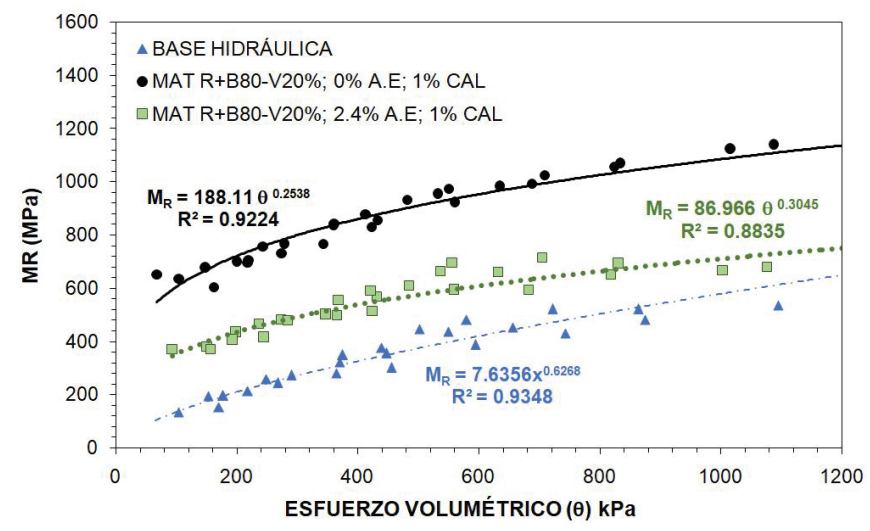

Figura 6. Módulo Resiliente para una base estabilizada con 2,4\% y $0 \%$ de asfalto espumado y $1 \%$ de cal vs base hidráulica convencional

Se puede observar que la base estabilizada con $2.4 \%$ asfalto espumado tiene valores superiores de $M_{R}$ con respecto a una base granular tradicional en todo el rango de esfuerzos volumétricos evaluados. Este aumento se puede asociar al material recuperado (RAP) el cual es un material que tiene una mayor rigidez que un material granular. Si se compara la mezcla reciclada con y sin asfalto espumado, se observa que la adición del asfalto espumado reduce los valores del $M_{R}$, esta reducción aumenta con el esfuerzo volumétrico. Siendo el aporte del asfalto espumado la mejora de la flexibilidad y cohesión, lo cual se traduce en una reducción en la rigidez de la mezcla de materiales (valor del $M_{R}$ ). Debido al incremento en los valores de $M_{R}$ de una base con material reciclado con respecto a una base hidráulica tradicional, se puede concluir que la condición más desfavorable del diseño será la pérdida de cohesión y no propiamente la capacidad de carga, ya que la inclusión del RAP, al ser un material más rígido, incrementa los valores de $M_{R}$.

\section{INFLUENCIA DE LOS PARÁMETROS DE DISEÑO.}

Con el objetivo de entender el comportamiento de los parámetros de diseño de la base estabilizada con asfalto espumado, se realizó un análisis del efecto del tipo de filler activo y porcentaje de asfalto espumado en la resistencia a la tensión indirecta, Cohesión, ángulo de fricción y Módulo Resiliente $\left(\mathrm{M}_{\mathrm{R}}\right)$.

\section{INFLUENCIA DEL FILLER ACTIVO}

La evaluación de la influencia del tipo de filler en los parámetros antes mencionados se presenta la Figura 7. Para el ensayo de resistencia a la tensión indirecta, la evaluación fue realizada tanto en condición de seca como en condición saturada y en tres diferentes contenidos de asfalto espumado. Para el caso del ensayo triaxial y ensayo módulo resiliente las evaluaciones se realizaron para un contenido de asfalto espumado de $2.4 \%$.

De los resultados obtenidos se puede observar que la mezcla con cal fue la que presentó menos susceptibilidad a la humedad. De igual forma se puede observar que la adición de un filler activo disminuye la susceptibilidad a la humedad de la mezcla, ya que en condición original la mezcla no resistía el acondicionamiento previsto en el ensayo. En las curvas tangenciales de los círculos de Mohr (Figura 7b) se puede observar que existe un cambio en el comportamiento del material con la adición de un filler activo (cambio de pendiente), siendo que la adición del filler aumenta la cohesión (2.2 veces para el cemento y 3.1 para la cal) y presenta una reducción del ángulo de fricción (20\% para el cemento y $12 \%$ para la cal). Los resultados obtenidos en el módulo resiliente (Figura 7c) muestran que al utilizar asfalto espumado se incrementa el valor de $M_{R}$ a bajos esfuerzos volumétricos $(\theta)$ en comparación de una base hidráulica tradicional. Para los materiales con filler activo el valor de $M_{R}$ incrementa en todo el rango de esfuerzos volumétricos, siendo que el valor de $M_{R}$ incrementa con el nivel del esfuerzo volumétrico mejorando su relación esfuerzo-deformación y por ende su capacidad estructural cuando forme parte de un pavimento. 
a)

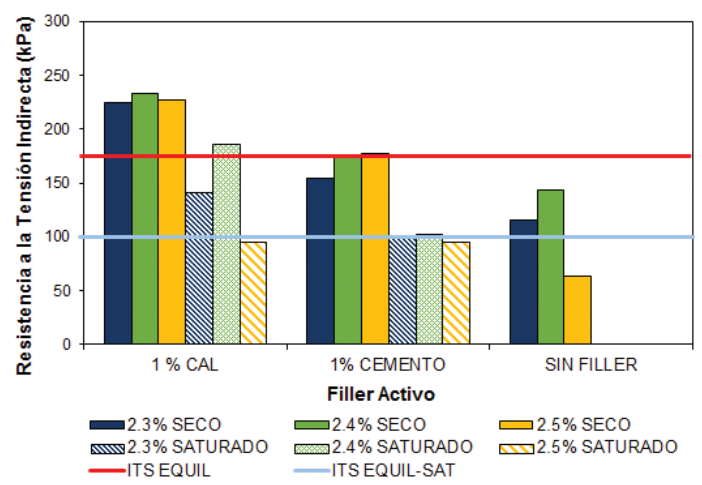

b)

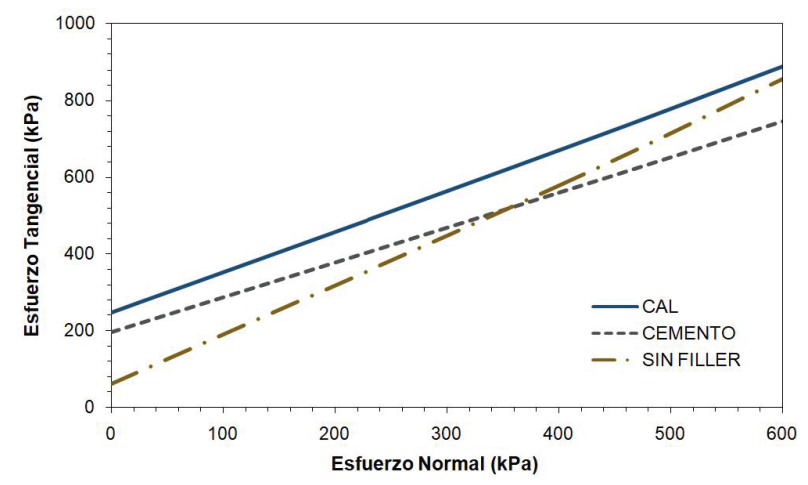

c)

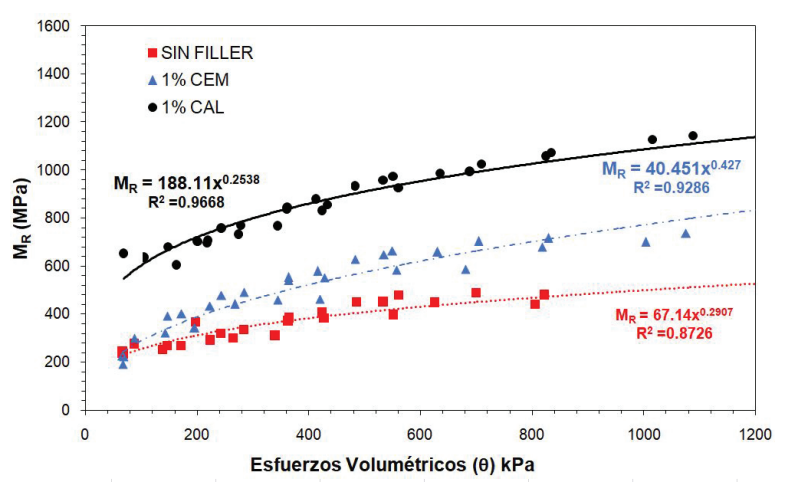

Figura 7. Influencia del filler en las propiedades mecánicas de la base estabilizada (+base)

\section{INFLUENCIA DEL CONTENIDO DE ASFALTO ESPUMADO}

La evaluación fue realizada con tres diferentes concentraciones de asfalto espumado (2.3\%, 2.4\% y 2.5\%). Para el caso del ensayo triaxial y ensayo módulo resiliente las evaluaciones se realizaron con $1 \%$ cal (Figura 8).

a)

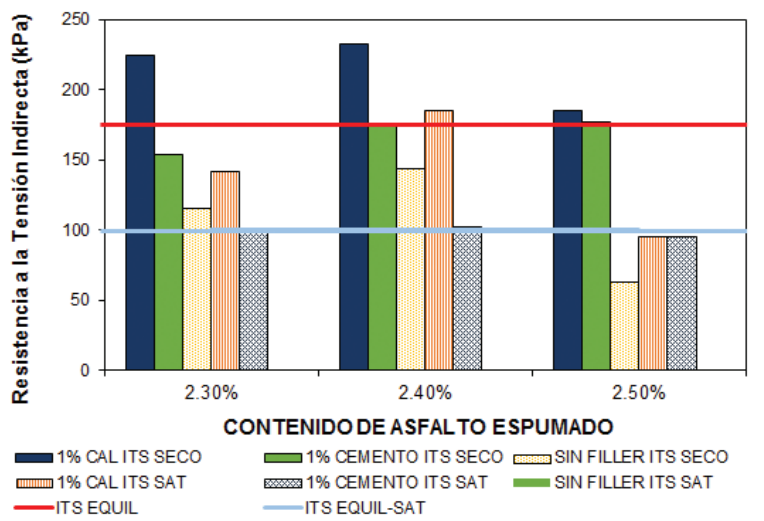

b)

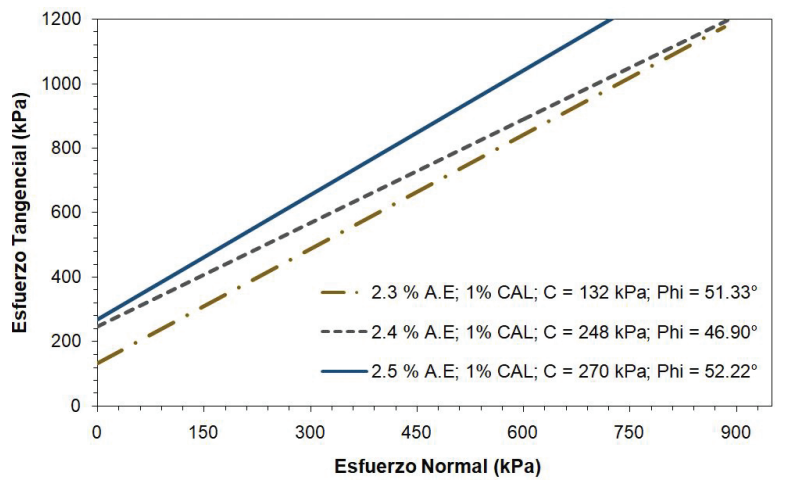

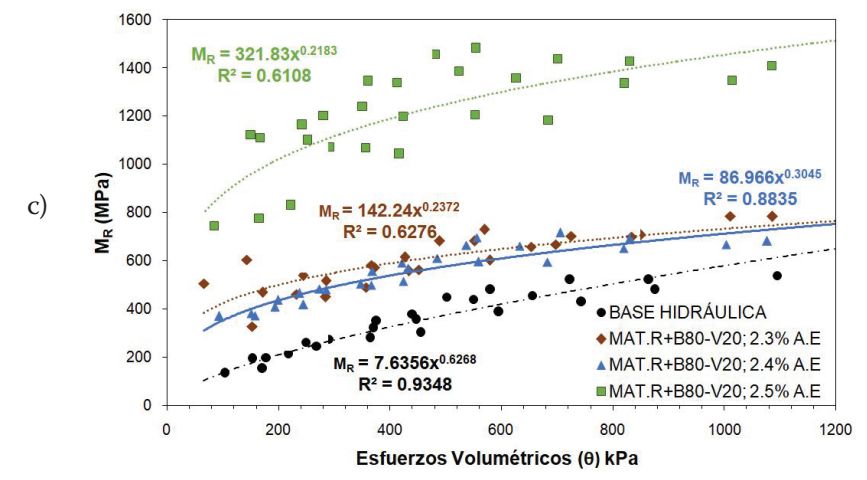

Figura 8. Influencia del contenido de asfalto espumado en las propiedades mecánicas de la base estabilizada 
De la Figura 8a es posible apreciar, que al aumentar el contenido asfalto espumado el valor $\mathrm{RTI}_{\text {Seco }}$ incrementa y posteriormente decae mostrando como valor óptimo el $2.4 \%$. No obstante, aun cuando los valores obtenidos se encuentren por encima del valor mínimo requerido, el desempeño de la mezcla sigue en función de los valores obtenidos de $\mathrm{RTI}_{\text {Saturado }}$. En las curvas tangenciales de los círculos de Mohr (Figura 8b) se puede observar que al incrementar el contenido de asfalto espumado se incrementa la cohesión de la base estabilizada, en cuanto al ángulo de fricción el comportamiento no tiene una tendencia clara. Los resultados muestran que para los contenidos de asfalto espumado de 2.3 y $2.4 \%$ los valores de $M_{R}$ son similares (Figura 8c), mientras que al aumentar el valor a $2.5 \%$ se observa un aumento significativo en el valor del $M_{R}$, lo cual indica que al adicionar un mayor porcentaje de asfalto espumado se mejora la capacidad de carga de la base estabilizada.

\section{COMENTARIOS Y CONCLUSIONES}

Existen diferentes metodologías para la estabilización de bases granulares, siendo el mayor beneficio de la estabilización con asfalto espumado la posibilidad de incorporar altos porcentajes de material reciclado (RAP) sin la necesidad de utilizar una fuente de calor. Esto también se podría realizar con una base estabilizada con asfalto convencional (base negra). Sin embargo, los porcentajes de asfalto serían superiores aumentando el costo de la estabilización, aunado al consumo de energía asociado a la fabricación de la mezcla de materiales.

El reto inicial en el diseño de la base estabilizada con asfalto espumado es definir una granulometría debido a que el material recuperado del pavimento (RAP) en general no cumple con los requerimientos establecidos en la metodología y es necesario agregar material granular de aporte. Por lo cual el procedimiento de obtención del RAP (escarificado-fresado de la carpeta) juega un rol fundamental en el porcentaje de material reciclado que se puede utilizar en una base estabilizada con asfalto espumado.

En la evaluación de la resistencia a la tensión indirecta, se pudo observar que los valores en condición seca son similares para cada una de las variantes analizadas, siendo la evaluación en condición saturada el parámetro de selección de la mezcla de materiales en una base estabilizada con asfalto espumado, por lo cual se le debe prestar especial atención en los procesos de diseño y control de calidad de este tipo de estabilizaciones.

Se pudo observar que con el aumento del contenido de asfalto espumado se reduce la susceptibilidad a la humedad, al igual que genera un aumento en la cohesión obtenida en el ensayo triaxial. Siendo que para concentraciones altas se presenta un aumento en el valor del $M_{R}$, cabe mencionar que el aumento de este parámetro aumenta el costo de la obra. Por lo cual, la selección adecuada del contenido de asfalto espumado se debe de realizar mediante un análisis costo-beneficio.

En cuanto a la adición del filler, se observó una mejora la cohesión del material, sin embargo, reduce la fricción de la base estabilizada. No obstante, la adición del filler incrementa notablemente los valores de $M_{R}$ en comparación con una base granular convencional. Para el caso particular de este estudio, el filler que obtuvo el mejor desempeño en los ensayos fue la cal.

Los valores de módulo resiliente obtenidos con las bases estabilizadas con asfalto espumado con RAP fueron superiores a los de una base granular. Este aumento está asociado a que el RAP tiene una mayor rigidez que el material granular. Por lo cual, se concluye que con la inclusión del RAP no se tendrá un problema en lo referente a la resistencia de la base estabilizada, lo cual asegura el desempeño siempre y cuando los criterios de cohesión y resistencia a la tensión indirecta en condición húmeda sean los adecuados.

\section{REFERENCIAS}

1. Abbas, A., Ali, A., Nazzal, M., Alhassan, A., Roy, A., \& Powers, D. (2013). Effect of temperature reduction, foaming water content, and aggregate moisture content on performance of foamed warm mix asphalt, 48, 1058-1066. Construction and Building Materials.

2. Austroads Ltd. (2015). Technical Report AP-T303-15. Design and Performance of Foamed Bitumen Stabilised Pavements: Progress Report 3. Sydney, Australia.

3. Dal Ben, M., \& Jenkins, K. J. (2014). Performance of cold recycling materials with foamed bitumen and increasing percentage of reclaimed asphalt pavement, 15:2, 348-371. Road Materials and Pavement Design.

4. Jenkins, K. J., Long, F. M., \& Ebels, L. J. (2007). Foamed bitumen mixes= shear performance?. International Journal of Pavement Engineering, 8(2), 85-98.

5. Marek, I., \& Anna, C.-K. (2013). Laboratory Study on Mechanical Parameters of FoamedBitumen Mixtures in the Cold Recycling Technology, 57. Modern Building Materials, Structures and Techniques.

6. Saleh, M. F. (2007). Effect of rheology on the bitumen foamability and mechanical properties of foam bitumen stabilised mixes. International Journal of Pavement Engineering, 8(2), 99-110.

7. Wirtgen. (2012). Wirtgen Cold Recycling Technology. Windhagen, Alemania: Wirtgen GmbH, Pages: 104-107. 